\title{
Orientaciones para la enseñanza de la escritura en la educación inicial y primaria en discursos oficiales y científicos. Colombia, 1994-2004'
}

\section{Prientation to writing teaching in early and elementary education on official and scientific speeches. Colombia, 1994-2004}

Fecha de recepción: 28 de marzo de 2008 Fecha de aceptación: 14 de julio de 2008
Isabel Borja Alarcón, Alfonso López Vega, Pilar Esther Méndez Rivera, Aldemar Segura Escobar, Flor Ängel Rincón

\section{Resumen}

Este artículo presenta una síntesis de la investigación titulada Orientaciones para la enseñanza de la escritura en la educación inicial y primaria en discursos oficiales y cientificos. Colombia, 1994-2004. La introducción del artículo permite la visualización total de dicho informe, luego, bajo el subtítulo desarrollo, se encuentran tres apartes dedicados a la síntesis de los supuestos teóricos utilizados para el análisis del objeto de estudio, las concepciones que a cerca de dicha enseñanza se expresaron a través de discursos oficiales y las orientaciones que para ella se revelan en los discursos científicos. También se presentan las conclusiones ofrecidas por la investigación y las referencias bibliográficas citadas.

\section{Palabras clave}

Enseñanza de la escritura, educación preescolar, educación primaria, Colombia.

\section{Summary}

This article provides a synthesis or overview of the research project entitled, "Approaches to the teaching of writing at foundation and primary level in official and scientific discourses, Colombia 1994-2004". It is composed of an introduction, which provides a general overview of the aforementioned paper, then, within the "Development" section, there are 3 subsections: 1) A section which examines the major recognised theories of the writing teaching, 2) A section which considers the theories which have been highlighted in official discourses; 3 ) and a section which introduces approaches of the scientific discourse to the teaching of writing. At the same time, the paper includes the conclusions contained in the research paper and the bibliography of this article.

1 La investigación que es fuente para este artículo fue financiada por el Centro de Investigaciones y Desarrollo Científico de la Universidad Distrital Francisco José de Caldas de Bogotá, D. C., Colombia. Los autores de este artículo son profesores de esta universidad e integrantes del Grupo de Investigación INTERTEXTO. 


\section{Key Words}

The teaching of writing, early years or initial writing, primary writing, Colombia.

\section{Introducción}

Distintas instancias reconocen la importancia de la escritura en el desarrollo de la actividad social; una muestra de dicho reconocimiento la ofrece la Declaración mundial sobre educación para todos, resultado de la Cumbre de representantes de los países reunidos en Jomtein (Tailandia) en 1990, la cual se refirió a la necesidad del dominio de la escritura para el logro del aprendizaje de nuevos conocimientos. En dicha declaración se afirma que "Saber leer y escribir constituye una destreza necesaria en sí misma y es la base de otras destrezas vitales"2.

En consonancia con la idea generalizada de la importancia de la escritura y considerando que el conocimiento acerca de cómo escribir es enseñable y aprendible, la sociedad asigna a la escuela, entre otras tareas, la enseñanza de la escritura ${ }^{3}$. Por los textos que nos hablan de la historia de dicha enseñanza, sabemos que ésta se constituyó en actividad escolar desde el surgimiento de la institución educativa y la vemos como preocupación social a través del tiempo. El reconocimiento social de la importancia de la escritura convoca a los académi$\cos$ y a los responsables de las políticas educativas de los distintos países a considerarla como objeto de reflexión, valoración y toma de decisiones. La Secretary's Commission on Achieving the Necessary Skills, US Departament of Labor ${ }^{4}$, por ejemplo, en

2 CONFERENCIA MUNDIAL DE EDUCACIONN PARA TODOS. (1a : 1990 : Jomtein) Declaración Mundial sobre Educación para Todos : La satisfacción de las necesidades básicas de aprendizaje. http://www.mineducacion.gov.co/cvn/1665/articles- 110467 archivo_pdf.pdf. p. 5.

3 Es preciso tener en cuenta que de esta tarea participa no sólo la escuela sino, también, otras unidades de la actividad social, entre ellas, la familia y los medios de comunicación.

4 SECRETARY'S COMMISSION ON ACHIEVING THE NECCESARY SKILLS US. DEPARTAMENT OF LABOR. EI 'know-how' (1991). Adaptado por Gómez Buendía, Hernando. En GÓMEZ BUENDÍA, Hernando (comp). Educación. La agenda su diagnóstico final de la indagación a empresarios, sindicatos, maestros y analistas del sector educativo estadounidense acerca de las necesidades educativas, alude a la formación en competencias fundamentales y laborales transversales; entre las primeras incluye las denominadas "habilidades básicas" y, como parte de estas, la escritura.

En el ámbito académico colombiano es posible identificar diversas concepciones acerca del desarrollo de la escritura; para los efectos de este estudio nos interesa centrar la atención en las concepciones de los niños que cursan los niveles de preescolar ${ }^{5} \mathrm{y}$ primaria de la educación formal; la lectura de varios documentos, que circulan tanto en la esfera científica como en la oficial, nos permite reconocer que no hay unidad de criterios con respecto a la valoración del dominio de la escritura que propicia la escuela. Como ejemplo de las concepciones diferentes que al respecto se han formulado, presentamos lo planteado por Pérez Abril, Banguero y Cisneros Estupiñán, quienes aluden a las dificultades en la adquisición de este dominio, y lo enunciado por Guzmán R., Correa Restrepo, Orozco Hormaza, Borja Alarcón y López Vega, cuya referencia a dicha adquisición señala logros importantes.

Pérez Abril ${ }^{6}$ alude a dificultades que los estudiantes de primaria y secundaria presentan para escribir e identifica, entre otras causas, los métodos y contenidos de enseñanza; así lo afirma:

\section{Problemática 1: No hay producción de textos, hay escritura oracional}

Hay una dificultad manifiesta en las producciones escritas de los estudiantes, tanto en educación básica

del siglo XXI. Bogotá : Programa de las Naciones Unidas para el Desarrollo. Tercer Mundo, 1996. p. 223.

5 Denominado para los efectos de este trabajo nivel inicial.

6 PÉREZ ABRIL, Mauricio. Leer y escribir en la escuela: algunos escenarios pedagógicos y didácticos para la reflexión. Bogotá : Instituto Colombiano para el Fomento de la Educación Superior, 2003. p. 11 
primaria como en secundaria, que consiste en la dificultad para elaborar textos completos, cerrados. La tendencia es a escribir oraciones o breves fragmentos.

\section{Posibles causas}

Hay una causa que se debe buscar en los primeros grados de la escolaridad, concretamente en los métodos de acercamiento a la escritura preescolar de los pequeños, en el primer grado de primaria o en el preescolar. Si la escuela utiliza métodos silábicos en los cuales la unidad de trabajo es la vocal, luego la sílaba, posteriormente la palabra y finalmente la frase, es esperable que la unidad de comunicación escrita que construye el niño esté referida a esas unidades.

Con respecto a la afirmación anterior, en nuestro criterio, es posible conceptuar que aunque la escuela proponga que el aprendizaje de la escritura siga la secuencia silaba-palabra-frase ${ }^{7}$, el niño tiene contacto con formas de la lengua escrita que le permiten superar los límites que implica dicha secuencia y esa superación ocurre en el salón de clases -pues el niño no es individuo aislado y pasivo frente a un profesor-y en otros ambientes en los que participa, en los cuales la lengua escrịta revela su carácter discursivo.

Por su parte, Banguero ${ }^{8}$ señala que en Colombia los niños: "Escriben textos muy breves, muy simples, su conocimiento está centrado en textos con contenidos escolares, dejando por fuera los textos indispensables en la interacción social". Esta autora no explica qué se entiende por "textos con contenidos escolares" ni a qué tipo de interacción social se refiere. En nuestro criterio, puede considerarse que los textos que llegan a la escuela y los que allí se producen están implicados en una forma específica en la interacción

7 No vocal-sílaba-palabra-frase porque, en castellano, toda vocal es una sílaba.

8 BANGUERO, Sandra P. Los niños necesitan maestros que lean y escriban bien. http://aupec.univalle.edu.co/informes/feb99/ lectura.html. social: la escolar, la cual da origen a discursos con características propias. Pero, mientras Banguero en la referencia indica como negativo que el conocimiento de textos esté centrado en los de contenido "escolar", Cisneros Estupiñán' señala como un problema que:

La asignatura de lenguaje, en muchos casos, se limita a la lectura lúdica de fábulas y cuentos, y la escritura, por mucho, a tratar de convertir a los niños en poetas y cuentistas con modelos que necesitan revisión; pero no se les presenta la posibilidad de entrar en relación con los textos argumentativos, explicativos, informativos, etc., lo cual perjudica enormemente su desempeño escolar y no proporciona al estudiante la motivación necesaria desde la asignatura de lenguaje ni desde las demás.

De esta cita puede inferirse que para Cisneros Estupiñán, en la referencia, la escuela se ocupa de textos lúdicos y literarios pero no de los argumentativos, explicativos e informativos que considera distintos a los lúdicos y literarios y de necesaria enseñanza. Con esto se evidencia su posición diferente a la de Banguero quien, como se observó, valora como negativo el que la institución educativa se haya centrado en los textos de "contenido escolar".

Al igual que en Banguero, ya referenciada, en Cisneros Estupiñán identificamos manifestaciones de prejuicio negativo hacia las producciones breves; adicionalmente, critica que la escuela propicie la escritura de cuentos y poemas, pero no textos argumentativos, explicativos e informativos; olvida esta autora que en un poema o en un cuento se pueden encontrar, como componentes textuales, argumentaciones, explicaciones y/o informaciones. Su referencia a que se enseña la escritura de cuentos y poemas con modelos que necesitan revisión, no aporta argumentos que sustenten su afirmación

9 CISNEROS ESTUPINAAN, Mireya. Mejorar los procesos lectoescriturales desde la educación básica. En La didáctica de la lengua materna : Estado de la discusión en Colombia. Santiago de Cali : Universidad del Valle, 2005. p. 218. 
por la falta de explicación acerca de cuáles son esos modelos y en qué consiste la revisión que requieren,.

En una dirección contraria a las dos inmediatamente aludidas, Guzmán R. ${ }^{10}$ reporta una experiencia de investigación en enseñanza de la escritura a niños de primero de primaria quienes, en el desarrollo de la misma, escribieron sobre diferentes temas y generaron textos de distintas tipologías (notas y artículos, noticias del periódico mural y cuentos), realizaron reflexiones semánticas y sintácticas, mejoraron el trazo de las letras, expresaron su comprensión de la función de los signos de puntuación, demostraron interés por escribir bien y aprecio por sus escritos. En este mismo sentido de valoración positiva del desarrollo del dominio de la escritura por niños, Correa Restrepo y Orozco Hormaza ${ }^{11}$ dan cuenta de su trabajo de investigación sobre el tema, en cuyas conclusiones se reconoce la importancia positiva de la actitud del profesor, su estilo de intervención y las modalidades del contrato enunciativo que conllevaron una experiencia de enseñanza en la que fueron tenidas en cuenta las estrategias de los niños para escribir y las del profesor para orientar el proceso. $\mathrm{Al}$ respecto escribieron:

El hallazgo más importante [...] fue haber podido realizar un trazado de las transformaciones graduales de las operaciones semióticas y discursivas y de los funcionamientos lingüísticos que dan cuenta de la intención y los conflictos de los personajes de los cuentos escritos por los niños.

[...] El análisis de los datos de los textos de la investigación, pone en evidencia que la gradua-

10 GUZMÁN R., Rosa Julia. El manejo del lenguaje escrito. En JURADO VALENCIA, Fabio Y BUSTAMANTE ZAMUDIO, Guillermo. Los procesos de la escritura : Hacia la producción interactiva de los sentidos. Santafé de Bogotá : Magisterio, 1998. p. 135-147.

11 CORREA RESTREPO, Miralba y OROZCO HORMAZA, Maria Cecilia. Dominio gradual de la intencionalidad en la escritura de textos narrativos. En Lectura y escritura para aprender a pensar. (2001 : Cartagena de Indias). Memorias del I Coloquio Internacional y III Regional de la Cátedra UNESCO para la lectura y la escritura en América Latina. Cali : Universidad del Valle, 2001. lidad de la intencionalidad es el resultado de una reorganización del funcionamiento cognitivo, que les permite a los niños proponer sujetos textuales dotados de estados mentales, que dan cuenta de los motivos de su acción.

En el sentido de ofrecer una visión positiva del trabajo de escritura en la escuela orientado durante la realización de un trabajo de investigación, Borja Alarcón y López Vega ${ }^{12}$, escribieron:

Entre los resultados, las observaciones de los profesores participantes en el sentido de que su trabajo [...] les permitió una nueva mirada sobre su hacer docente y, en particular, posibilitó la reflexión acerca de cómo son y cómo deben ser sus comunicaciones en la escuela. La apertura en el campo comunicativo ha permitido la creación de hermosos textos que todos, profesores y estudiantes, han disfrutado [...] diálogos, espacios donde se comparta la palabra sin temor $[\ldots]$ textos formales e informales.

Señalamos una diferencia importante entre las afirmaciones que reportan los desempeños en escritura como negativos y las que, por el contrario, los valoran como positivos: las primeras son generales, sin identificación de población específica y sin que los investigadores se enuncien como participantes de la experiencia que conlleva al concepto negativo; las segundas, identifican la población a la que se refieren y los investigadores se presentan involucrados en la actividad que revela el dominio positivo de la escritura.

Desde nuestra comprensión, las afirmaciones que indican dificultades en la escritura de los niños pueden tener un mayor o menor impacto en el ámbito académico, dependiendo de quienes las formulen. Así,

12 BORJA ALARCON, Isabel y LOPEZ VEGA, Alfonso. Las palabras en la escuela. Experiencia educativa en el área de castellano. Santafé de Bogotá : Universidad de los Llanos, 1999. p. 144. 
por ejemplo, lo indicado por Llinás ${ }^{13}$ con respecto a la calidad de la educación en Colombia:

Baja calidad de la educación básica, sobre todo la oficial y la de gran parte de los colegios de estrato medio y bajo. Una indicación aunque parcial, de la baja calidad es el deficiente logro de los objetivos de aprendizaje, tal como fue establecido en el tercero y quinto grados por los estudios el Sistema Nacional de Evaluación de la Calidad (SABER). En particular, se señala como problema crítico las deficiencias en las competencias básicas en el dominio de la lectura y la escritura y en el desarrollo del pensamiento lógico matemático.

El anterior aparte de Llinás ha servido de antecedente para la comprensión de que en Colombia los estudiantes de primaria tienen problemas con la escritura; ello, porque la afirmación la hace un académico reconocido que señala como base de su enunciación los resultados de las pruebas SABER, que en Colombia se aplican desde 1991 aunque con intervalos de tiempo no regulares. No obstante, la certeza que se podría tener frente a la afirmación de Llinás, con respecto a que las pruebas en mención demuestran insuficiente dominio de la escritura, se desmorona cuando se lee lo que el Instituto Colombiano para el Fomento de la Educación Superior (ICFES) responde frente a la pregunta de cómo se evalúa en lenguaje: "La prueba [SABER] está compuesta por preguntas de opción múltiple con única respuesta, que corresponden al objeto de la evaluación: el proceso de lectura ${ }^{14}$. Es decir, el ICFEs señala que no se evalúa el dominio de la escritura.

Podemos considerar como una reiteración de que las pruebas en mención no evalúan la escritura,

13 LLINÁS, R. R. "El reto. Ciencia, educación y desarrollo: Colombia en el siglo XXI". En Misión de ciencia, educación y desarrollo. Colombia : al filo de la oportunidad. Informe conjunto. Santafé de Bogotá : Magisterio, 1995. p. 75.

14 INSTITUTO COLOMBIANO PARA EL FOMENTO DE LA EDUCACIÓN SUPERIOR. La evaluación en lenguaje. http://200.14.205.63:8080/portalicfes/ home_2/rec/arc_3595.pdf. p. 7. lo afirmado por la Oficina Asesora de Planeación y Finanzas del Ministerio de Educación Nacional $(\mathrm{MEN})^{15}$ al dar cuenta de los resultados obtenidos por los estudiantes de quinto de primaria en las pruebas SABER:

Las competencias de lenguaje hacen énfasis en la capacidad de comunicarse: hablar con calidad, escribir y comprender lo que se lee. Se pasa de comprender textos sencillos a hacer inferencias, comparar textos y hacer interpretaciones críticas. Igualmente, implica destrezas y conocimientos para usar nuevos medios de comunicación como televisión, radio e Internet, así como los lenguajes del teatro, la música y la expresión corporal. Por esto la evaluación censal se centró en la comprensión lectora, que abarca la utilización del lenguaje para comunicarse, la comprensión de diferentes tipos de textos, la comunicación escrita y la relación entre distintos textos.

El enunciado antes citado ratifica que no se evalúa escritura en las pruebas SABERy alude a que la comprensión de lectura implica la comunicación escrita, pero no asume la discusión que clarifique que participar en la comunicación escrita como lector exige características distintas a la participación como escritor. Esto equivale a decir que en el enunciado se alude a la comunicación escrita como articulada a la lectura de textos escritos, no a su producción.

Las dificultades para la evaluación de la escritura, tanto en pruebas censales como muestrales, nacionales e internacionales, por fuera de la actividad escolar de desarrollo curricular, parecen ser una constante, perceptible a través de la verificación de que, aún en los casos en que se ha partido del propósito de evaluarla, no se reporta valoración del dominio alcanzado en la elaboración de textos escritos por los estudiantes evaluados. Otra prueba de esto es lo

15. COLOMBIA. MINISTERIO DE EDUCACIONN NACIONAL. OFICINA ASESORA DE PLANEACION Y FINANZAS. EI desarrollo de la educación en el siglo XXI. Informe Nacional de Colombia. Bogotá : http://www.oei.es/quipu/colombia/ ibecolombia.pdf, junio de 2004. p. 23. 
escrito por Casassus ${ }^{16}$ quien informa, con respecto a las pruebas aplicadas a niños de primaria por el Estudio internacional comparativo sobre lenguaje, matemática y factores asociados realizado por el Laboratorio Latinoamericano de Evaluación de la Calidad de la Educación que:

Para la construcción de las Pruebas se definió entonces que los aspectos a observar serían la lectura y la escritura como formas de hacer, en las que el estudiante pone en juego su "saber-saber-hacer" [...] con el Lenguaje. Por tal motivo, y limitada también por las características de una prueba de aplicación masiva, la evaluación realizada tuvo como objeto inicial el proceso de comprensión lectora y el proceso de producción escrita. No obstante lo anterior, el análisis de resultados presentado en este Capítulo se basa solamente en el proceso de comprensión lectora.

De conformidad con Casassus, aunque se diseñó la prueba para evaluar lectura y escritura, sólo se tienen en cuenta y se reportan los resultados de comprensión de lectura; sin embargo, no se informa por qué no se ofrecen los resultados de la prueba de escritura.

Lo que hasta ahora parece ser es que las afirmaciones acerca de los deficientes logros en escritura de los niños colombianos en pruebas estatales no tienen fundamento en reportes oficiales y que afirmaciones como las enunciadas por Banguero en los términos de que: "Evaluaciones realizadas a nivel nacional han descubierto que el desempeño de lectura y escritura de los niños colombianos es muy deficiente" 17 , por su carácter de generalidad -no indica en cuáles pruebas- pueden carecer de validez científica.

16 CASSASUS, Juan (coord.). Primer estudio internacional comparativo sobre lenguaje, matemática y factores asociados, para alumnos del tercer y cuarto grado de la educación básica. Informe técnico. Laboratorio Latinoamericano de Evaluación de la Calidad de la Educación -UNESCO- http://unesdoc.unesco. org/images/0014/001492/149268s.pdf, 2001. p. 21.

17 CASSASUS, Juan (coord.). Ibid.
Esa inconsistencia entre las voces de la esfera oficial, que señalan la no evaluación en escritura, y la esfera científica -que aluden a resultados deficientes obtenidos por los estudiantes evaluados-, puede afectar de una manera negativa la enseñanza de la escritura en la escuela porque menoscaba la imagen del niño como escritor y la del profesor como persona que orienta los procesos escolares de aprendizaje.

Por la influencia que las orientaciones oficiales y cientificas ejercen en la actividad escolar, consideramos necesario clarificar cuáles orientaciones se formularon para la enseñanza de la escritura en Colombia, en los grados de preescolar y de primaria, de tal manera que se observe la relación entre los enunciados de los discursos emanados pues, de lo contrario, no se reconocerá cuál es la base conceptual de las formulaciones que desde la academia y la política educativa se pronuncian con respecto a la escritura y su enseñanza y cuáles son los puntos de confluencia y los de contradicción y, en consecuencia, se puede continuar generando discursos incoherentes acerca de la enseñanza de la escritura en Colombia sin hacer explícitos los elementos que implican dicha incoherencia.

Nuestro interés en observar los enunciados científico y oficial acerca de la enseñanza de la escritura en la educación preescolar y primaria en la década comprendida entre 1994 y 2004 obedece a que en 1994 el gobierno expidió la Ley General de Educación, la norma educativa más importante que, para los niveles que anteceden al universitario, ha promulgado el gobierno en desarrollo de la Constitución Política Nacional de 1991 y que ha dado origen a una renovación en las distintas esferas de la actividad en nuestro país. La investigación comprende hasta 2004 para tener oportunidad de observar el desarrollo de la política educativa y de los enunciados científicos con respecto a la enseñanza de la escritura en la educación preescolar y primaria durante una década.

Por todo lo anterior, nos planteamos el problema de investigación que expresamos en la siguiente pre- 
gunta ¿Qué características revelan las orientaciones para la enseñanza de la escritura en la educación preescolar y primaria en los discursos colombianos, oficial y científico, producidos entre 1994 y 2004 ?

Para dar respuesta al problema de investigación, nos aproximamos a la literatura especializada que ofrece reflexiones teóricas acerca de: 1) la escritura en el marco de la realización de la discursividad en las distintas esferas de la actividad humana; 2) la significación del término 'escritura'; 3) la enseñanza como actividad que contribuye a la formación en escritura; 4) el proceso didáctico como marco para la comprensión de la enseñanza de la escritura. En lo referente a la escritura como realización de la discursividad, consultamos, entre otros, los trabajos de Jaimes Carvajal ${ }^{18}$, Shishkova y Popok ${ }^{19}$, van Dijk $^{20}$, Rosental e Iudin ${ }^{21}$, Aguirre ${ }^{22}$, Geymonat ${ }^{23}$ y De Mora $^{24}$; en lo atinente a la significación de 'escritura', el estudio se centró, principalmente, en lo enunciado por Spirkin ${ }^{25}$, Gorski ${ }^{26}$, Bloomfield ${ }^{27}$,

18 JAIMES CARVAJAL, Gladys. El lenguaje y la comunicación humana. En JAIMES CARVAJAL, Gladys (coord.). Comprensión y producción de textos orales y escritos en el nuevo sistema acusatorio penal. Bogotá : Consejo Superior de la Judicatura, 2006.

19 SHISHKOVA, T. H. y POPOK, V. K. L. Estilística funcional. (s. l.) : (s. e.), 1989.

20 VAN DIJK, Teun. A. Ideología : Una aproximación multidisciplinaria. Traducción de Lucrecia Berrone de Blanco. Barcelona : Gedisa, 2000.

21 ROSENTAL, M. e IUDIN, P. Diccionario filosófico. Traducción Editora Política. (s. c.) : Combinado poligráfico de Guantánamo 'Juan Marinello', 1975.

22 AGUIRRE, Mirta. En torno a la expresión poética (1971). En Dubsky, J. y otros. Selección de lecturas para redacción. La Habana : Pueblo y Educación, 1987. p. 129-156.

23 GEYMONAT, L. El pensamiento científico. Traducción de José Babini. 4 ed. Buenos Aires : Editorial Universitaria de Buenos Aires, 1969.

24 DE MORA, J. M. Milenios de atracción entre la transmisión oral y la escrita. En India. Acta poética 26 (1-2). Primavera-otoño, 2005.

25 SPIRKIN, A. G. Origen del lenguaje y su papel en la formación del pensamiento. En Gorski, D. P.y otros. Pensamiento y lenguaje. Traducción de Augusto Vidal Roger. México : Grijalbo, 1961.p. 9-67.

26 GORSK1, D. P. Lenguaje y conocimiento En Gorski, D. P. y otros. Pensamiento y lenguaje. Traducción de Augusto :Vidal Roger. México: Grijalbo, 1961.p. 8-105.

27 BLOOMFIELD, Leonard. Lenguaje. Traducción Universidad
De Saussure ${ }^{28}$, Slakta ${ }^{29}$, van Dijk ${ }^{30}$, Ferreiro ${ }^{31}$ y Tolchinsky Landsmann ${ }^{32}$. Para la comprensión de la enseñanza como propiciadora de la formación en escritura, la atención se dedicó a los escritos de Duckworth ${ }^{33}$, Garton y Pratt ${ }^{34}$, Tolchinsky y Simó ${ }^{35}$, Jolibert, el Grupo de docentes de ECOUEN $^{36}$, GoODMAN $^{37}$ Y ARMSTRONG $^{38}$, ENTRE OTROS; LA REFLEXIÓN ACERCA DEL PROCESO DIDÁCTICO, CONSULTÓ LOS PLANTEAMIENTOS DE Althusser ${ }^{39}$, Bourdieu y Passeron ${ }^{40}$ y Álvarez de ZAYAS, fundamentalmente.

Nacional Mayor de San Marcos. Lima, Perú : Universidad Nacional Mayor de San Marcos, 1964.

28 DE SAUSSURE, Ferdinand. Curso de lingüistica general. Madrid : Akal, 1980.

29 SLAKTA, Denis. L'ordre du texte En Étude de linguistique appliquée. $\mathrm{N}^{\circ} 19$. Centre d'études du français moderne et contemporain. Paris : Didier, 1975. p. 30-42.

30 VAN DIJK, Teun A. Estructuras y funciones del discurso. Bogotá : Siglo XXI, 1980.

31 FERREIRO, Emilia. Alfabetización : Teoría y práctica. 4 ed. México : Siglo XXI, 2001.

32 TOLCHINSKY LANDSMANN, Liliana. Aprendizaje del lenguaje escrito: Procesos evolutivos c implicaciones didácticas. Barcelona : Anthropos, 1993.

33 DUCKWORTH, Eleonor. Lenguaje y pensamiento según Piaget. En Coll, C. (comp). Psicología genética y educación : Recopilación de textos sobre las aplicaciones pedagógicas de las teorías de jean Piaget. Barcelona : Oikos-Tau, (s. f.). p. 61-74.

34 GARTON, Alison y PRATT, Chris. Aprendizaje y proceso de alfabetización : El desarrollo del lenguaje hablado y escrito. Traducción de Magda Rivero. Barcelona: Centro de Publicaciones del Ministerio de Educación y Ciencia, 1991.

35 JOLIBERT, Jossethe. y Grupo de docentes de ECOUEN. Formar niños productores de textos. $6 \mathrm{ed}$. Traducción de Viviana Galdames Franco y Alejandra Medina Moreno. Santiago de Chile : Dolmen, 1995.

36 GOODMAN, Kenneth. El aprendizaje y la enseñanza de la lectura y la escritura. En Revista Enunciación $N^{\circ} 8$. Bogotá : Universidad Distrital Francisco José de Caldas, agosto. 2003. p. 77-98.

37 ARMSTRONG, Thomas. Inteligencias múltiples en el aula : Guía práctica para educadores. Traducción de Remedios Diéguez. Barcelona : Paidós Ibérica, 2006.

38 ALTHUSSER, Louis. Sobre la ideología y el Estado. En Escritos. Barcelona : Laia, 1974.

39 BOURDIEU, Pierre. y PASSERON, J. C. La reproducción : Elementos para una teoría del sistema de enseñanza. México : Fontamara, 1998.

40 ÁLVAREZ DE ZAYAS, Carlos M. La escuela en la vida: Didáctica. 3 ed. La Habana : Pucblo y Educación, 1999 
La identificación de las características del objeto de estudio también se posibilitó mediante la aproximación a la realidad de la enseñanza de la escritura preescolar y primaria entre 1994 y 2004 a través de: 1) documentos estatales; 2) trabajos de investigación colombianos publicados en libros y revistas, reportes de investigación realizados en opción a título profesional y de posgrado y manuales escolares colombianos para la enseñanza preescolar y primaria de castellano.

De conformidad con lo anterior, el objeto de esta investigación se basó en las orientaciones para la enseñanza de la escritura en la educación preescolar y primaria. El campo de acción, las orientaciones para la enseñanza de la escritura en la educación preescolar y primaria en Colombia, formuladas en los discursos científico y oficial entre 1994 y 2004. El objetivo, identificar las orientaciones que para la enseñanza de la escritura en la educación preescolar y primaria se formularon en los discursos cientifico y oficial colombianos producidos entre 1994 y 2004.

Para la obtención del objetivo fue necesario dar respuesta a las siguientes preguntas de investigación:

- ¿Qué fundamentos teóricos sustentan el análisis de las orientaciones para la enseñanza de la escritura en la educación preescolar y primaria?

- ¿Qué criterios sobre la enseñanza de la escritura en la educación preescolar y primaria se puede identificar en discursos oficiales colombianos producidos entre 1994 y 2004 ?

- ¿Qué criterios acerca de la enseñanza de la escritura en la educación preescolar y primaria en Colombia, entre 1994 y 2004 , es posible reconocer en discursos de la esfera científica?

- ¿Qué relaciones hay entre las orientaciones formuladas, entre 1994 y 2004, en los discursos científico y oficial colombianos acerca de la ense- ñanza de la escritura en la educación preescolar y primaria?

Para dar respuesta a las anteriores preguntas de investigación, se cumplieron las siguientes tareas:

- Identificación de los criterios teóricos que sustentan el análisis de las orientaciones para la enseñanza de la escritura en la educación preescolar y primaria.

- Reconocimiento de las orientaciones que para la enseñanza de la escritura en la educación preescolar y primaria colombianas, en el periodo citado, expresaron los discursos oficiales.

- Comprensión de los criterios que acerca de la enseñanza de la escritura en la educación preescolar y primaria en Colombia, en el lapso de tiempo señalado, expresaron los discursos científicos.

- Identificación de la relación entre las orientaciones que acerca de la enseñanza de la escritura en la educación colombiana, en los niveles de preescolar y primaria, se formularon entre 1994 y 2004 en los discursos científico y oficial.

En la realización del trabajo se utilizaron los siguientes métodos:

- Análisis-síntesis e inducción-deducción, en la recopilación y procesamiento de la información.

- Sistémico-estructural, en la articulación de los elementos que permitieron la comprensión de las orientaciones para la enseñanza de la escritura en la educación preescolar y primaria en los discursos científico y oficial colombianos entre 1994 y 2004.

- Histórico-lógico, en la identificación de las concepciones acerca de la enseñanza de la escritura. 
- Estudio documental, en el análisis de los documentos escritos -éste se aplicó de conformidad con lo señalado por Bravo Sierra ${ }^{41}$.

- Comparativo, en el estudio contrastivo entre los datos obtenidos a partir del estudio de las orientaciones para la enseñanza de la escritura en los discursos científico y oficial colombianos.

Puesto que este trabajo se ocupa de las orientaciones oficiales y científicas para el proceso didáctico de la escritura, las categorías de análisis son los componentes de dicho proceso; para su definición seguimos lo planteado por Álvarez de Zayas ${ }^{42}$.

Para la obtención de la información se analizaron 60 manuales escolares de castellano editados por diferentes casas colombianas para su uso en nuestro país y 102 monografías de grado de aspirantes a título de las universidades Distrital Francisco José de Caldas, Pedagógica Nacional y Nacional de Colombia, de Bogotá. Ambas muestras -de manuales y de monografías- fueron seleccionadas intencionalmente.

El aporte teórico de este trabajo consiste en que, a la luz de los desarrollos científicos, se conceptúa la realidad de las orientaciones que para la enseñanza de la escritura en la educación preescolar y primaria fueron formuladas en los discursos científico y oficial colombianos entre 1994 y 2004. La significación práctica está dada en la caracterización de las orientaciones para la enseñanza de la escritura en la educación preescolar y primaria en Colombia entre 1994 y 2004 que explicita la relación entre los discursos científico y oficial; esta caracterización puede servir de base para una mejor comprensión de dichas orientaciones y de su interrelación y, a partir de ella, realizar estudios comparativos sobre el mismo objeto a partir de discursos de otras esferas de la actividad que, junto con este, sirvan de base para la elabora-

41 BRAVO SIERRA, R. Investigación social. Madrid : Paraninfo, 1996.

42 ÁLVAREZ DE ZAYAS. Op. cit. ción de nuevas propuestas para el desarrollo de la enseñanza de la escritura en la educación preescolar y primaria colombianas.

La novedad científica de este trabajo radica en que articula componentes implicados en las orientaciones para la enseñanza de la escritura en la educación preescolar y primaria en Colombia entre 1994 y 2004 que en análisis anteriores no habían sido articulados; de esta forma, se propone una perspectiva que refleja dicha realidad advirtiendo su complejidad.

El reporte de la investigación está estructurado en: introducción, dos capítulos, conclusiones, recomendaciones, bibliografia y anexos. En la introducción, se expone el problema de investigación, los antecedentes teóricos y el diseño de la investigación. El primer capítulo, que constituye el marco teórico, se ocupa de la referencia a la discursividad y su relación con la actividad, analiza diferentes acepciones del término 'escritura', reflexiona acerca de la importancia de la enseñanza en el desarrollo de las capacidades para la escritura y explicita la teoría didáctica de corte internacional a la que se recurre para el análisis del objeto de estudio. En el segundo capítulo, que revela la aproximación a la realidad colombiana, se consideran las concepciones acerca de la enseñanza de la escritura en la educación preescolar y primaria en discursos oficiales y científicos producidos entre 1994 y 2004.

\section{Desarrollo}

Este aparte comprende tres componentes. En el primero, se expresan los elementos teóricos que sirven de base para analizar nuestro objeto de estudio; en el segundo, se dan a conocer los resultados de la indagación acerca del objeto de estudio, en discursos colombianos de la esfera de la actividad oficial; en el tercero se presentan los resultados de la investigación acerca de las orientaciones para la enseñanza de la escritura en discursos científicos colombianos: textos publicados por especialistas, 
trabajos de grado profesional y de posgrado -especialización- $\mathrm{y}$ manuales escolares.

Criterios teóricos para el análisis de las orientaciones para la enseñanza de la escritura en la educación inicial y primaria

En este capítulo se propone analizar el objeto de estudio en investigación teniendo en cuenta los planteamientos que implica la comprensión de la escritura articulada a las distintas esferas de la actividad que la han constituido y en las cuales se desarrolla. Se da cuenta de que la literatura certifica considera el texto como unidad de escritura, constituido en discurso en virtud de la dinámica comunicativa y conformado por niveles que son factibles de análisis con base en el conocimiento acerca de la actividad discursiva.

Por el gran impacto que ejerce en las formulaciones acerca de la adquisición de la escritura por los niños, se reflexiona sobre la comprensión, expresada por distintos teóricos, de que los niños, antes de ingresar a la institución escolar, tienen aproximación al conocimiento de la lengua escrita. Se señala, así mismo, que a partir del dominio inicial, que, en nuestro criterio implica la interiorización del carácter arbitrario y convencional de la escritura, el niño continúa con la apropiación del código escrito para su uso en diversas circunstancias. Con respecto a la pregunta por cómo continúa el niño esta adquisición del dominio de la escritura, se presentan tres formas distintas de responder, dependiendo de cuál es el centro de atención (el texto, el proceso o el escritor).

En este acápite, también se ofrece la reflexión acerca del proceso didáctico y sus componentes, a partir del criterio de que la escritura es objeto de enseñanzaaprendizaje.
Orientaciones para la enseñanza de la escritura en los discursos oficiales colombianos entre 1994 y 2004

En este acápite interesa tener en cuenta los componentes humanos del proceso de enseñanza aprendizaje y los componentes propiamente didácticos.

En lo que respecta a los componentes humanos, los criterios para la enseñanza de la escritura, expresadas en los discursos oficiales colombianos entre 1994 y 2004, reconocen la importancia del profesor como orientador del trabajo didáctico y del estudiante como participante activo y centro del proceso de formación.

Acerca de los componentes propiamente didácticos, en los discursos oficiales el énfasis está dado a los objetivos, la forma del proceso y los materiales didácticos. En lo que respecta a los objetivos de la enseñanza de la escritura en el preescolar la Ley General de Educación ${ }^{43}$ entra en contradicción con la Resolución $N^{\circ} 2343$ del 5 de junio de 1996: la Ley implica el aprestamiento, la Resolución alude a la pertinencia del dominio de formas no convencionales de escritura; la contradicción radica en que para obtener el dominio de formas no convencionales no se requiere el aprestamiento sino la atención a la escritura como actividad significativa socialmente. En cuanto a la forma de organización del trabajo didáctico, el Estado orienta la modalidad de pedagogía por proyectos. Destacamos como muy importante el compromiso estatal de dotar las instituciones de materiales didácticos producidos por las instituciones estatales y privadas, pero los mismos documentos oficiales revelan que esta política no se ha llevado a la realidad.

El Estado expresa la decisión de propiciar la investigación científica como vía para superar los problemas educativos, con ello, en nuestro criterio,

43 Ley General de Educación, Artículo 16. "Objetivos específicos de la educación preescolar. $[\ldots]$ b). El crecimiento armónico y equilibrado del niño, de tal manera que facilite la motricidad, el aprestamiento y la motivación para la lecto-escritura". 
visualiza el camino pertinente; sin embargo, como él mismo lo indica en sus documentos, el Programa de Investigación del Plan Decenal de Educación no se cumplió.

Orientaciones para la enseñanza de la escritura en los discursos científicos colombianos entre 1994 y 2004

Para las consideraciones que nos ocupan en este aparte, la disertación se refiere, primero, a las orientaciones que implican los componentes humanos de la actividad didáctica y, luego, las relacionadas con los componentes propiamente didácticos del proceso de formación escolar.

En cuanto a los sujetos implicados en el proceso, hay una marcada atención al profesor de quien prevalece una imagen negativa, asociada al predominio de un señalamiento negativo de sus características para el desempeño profesional y se ofrece una imagen positiva del estudiante como reconocimiento de sus características personológicas.

El análisis de los textos relacionados con la enseñanza de la escritura que, entre 1994 y 2004, publicaron los especialistas revela que, en su comprensión de la escritura, siguen los postulados de distintos teóricos reconocidos internacionalmente; se observa la tendencia a considerar la escritura como una competencia comunicativa. La necesidad social de que los niños aprendan a escribir se entiende como respuesta a los requerimientos del trabajo escolar y de la evaluación estatal, principalmente. Tanto los objetivos de la formación, como los contenidos, los métodos y la evaluación se presentan, en un número significativo de los trabajos de los especialistas, de una manera no precisa.

En los trabajos de grado profesional y de posgrado -especialización-, se considera que el docente es el orientador del proceso didáctico, que el estudiante es un participante activo y que la familia una colabo- radora en el proceso; estos participantes se revelan con sus características y en su función en el marco de la didáctica de la escritura. En estos trabajos se reconoce que la escritura es importante para responder a los requerimientos escolares; se determinan los objetivos de la formación a partir de un trabajo de diagnóstico cuyos resultados establecen los contenidos, los procedimientos metodológicos, la forma de organización del proceso, los materiales didácticos y la evaluación; estos componentes del proceso didáctico se identifican de manera precisa. En lo metodológico, predomina el método de búsqueda parcial o heurística, el proceso adopta la forma de trabajo individual y grupal para la realización de talleres, se recurre a diversos materiales y las propuestas de evaluación se encuentran en consonancia con los contenidos.

En lo que se refiere a las propuestas para la enseñanza de la escritura en manuales escolares de preescolar y primaria, en cuanto a los componentes humanos del proceso didáctico, hay predomino de la imagen del estudiante la cual, en la mayoría de los casos, es positiva, tanto en lo personológico como en su desempeño; hay escasa alusión a los docentes y el ejercicio profesional se observa asociado al género femenino, predominantemente. El estudiante se presenta como llevando la iniciativa del proceso a partir del cumplimiento de las indicaciones del libro el cual se constituye en el principal "interlocutor" del niño durante el proceso didáctico.

En general, los autores no especifican sus concepciones acerca de la escritura ni de la necesidad que implica su enseñanza. Los autores acogen los objetivos de la formación que indican los documentos estatales y redactan otros que estiman pertinentes; estos se encuentran asociados a los indicadores de logros y a los estándares de competencias. Los contenidos abarcan desde actividades de aprestamiento hasta el trabajo con distintos tipos de discursos; en casi todos los textos se propone el aprendizaje de la escritura de distintos textos, propios de las diversas esferas de la actividad sin considerar en cuáles tiene 
lugar la discursividad del niño y, además, en general, se revela la necesidad de ajustar lo contenido al desarrollo científico; lo mismo ocurre con la metodología que se propone. La forma de organización del trabajo muchas veces no se expresa de manera comprensible, aunque predomina el trabajo individual y por proyectos. En lo que respecta a la evaluación, se puede señalar que no hay propuestas claras acerca de cómo realizarla y hacia dónde debe conducir.

\section{Conclusiones}

- El análisis de las características de las orientaciones para la enseñanza de la escritura implica la determinación de un marco teórico que lo sustente. En nuestro criterio, la teoría acerca de la discursividad en las distintas esferas de la actividad humana revela su carácter científico, por lo que es posible recurrir a ella para dicho análisis ya que la escritura se genera en dichas esferas y recibe la influencia de ellas.

- El estudio de las posiciones teóricas que expresan los discursos oficiales y los científicos -con excepción de los trabajos de grado- acerca de la enseñanza de la escritura en la educación preescolar y primaria en Colombia, entre 1994 y 2004, revela la carencia de un marco teórico que oriente las formulaciones que se elaboran con respecto a todos los componentes del proceso didáctico en estudio; como consecuencia de esto:

\section{En los discursos oficiales:}

- Los componentes humanos del proceso didáctico se conciben, en algunos documentos, como revelando las características requeridas para su desempeño; pero en otros como carentes de ellas.

- En lo que respecta a los componentes propiamente didácticos, las directrices se enuncian de manera general y es posible encontrar ejemplos de orientaciones que entran en contradicción.
- Se enuncian políticas cuyo cumplimiento podría contribuir a superar las contradicciones que se observan en el discurso oficial, pero éstas se han quedado, en la práctica, en enunciados, tal como sucede con la investigación educativa y la elaboración y distribución de materiales didácticos por parte de las instituciones y las entidades regionales en asocio con la empresa privada.

\section{En los discursos científicos}

- Discursos publicados por los especialistas: Las imágenes de los componentes humanos del proceso didáctico permite observarlos, en ocasiones, como cumpliendo los requisitos que se esperan para su desempeño $y$, en otras, como sin esos requisitos. Hay una marcada tendencia a mostrar que el profesor tiene características negativas en oposición al estudiante quien aparece, ante todo, como mejor dispuesto para dicho proceso. Los componentes propiamente didácticos del proceso se expresan de manera general.

- En los trabajos de aspirantes a título profesional y de posgrado-especialización. Hay un número significativo de este tipo de textos en donde se reconoce la importancia social de la escritura, se identifican los componentes humanos del proceso como revelando las características pertinentes para el ejercicio del trabajo y se especifican claramente todos los componentes propiamente didácticos de la actividad.

- En los manuales escolares. En cuanto a los componentes humanos implicados, el profesor se muestra como casi ausente del proceso, mientras el estudiante es la persona principal, marcada como aquel a quien el texto dirige la palabra. En las pocas representaciones de docentes se observa el predominio de personas del género femenino. En lo que respecta a los componentes propiamente didácticos del proceso, hay dificul- 
tades en su explicitación, siendo la principal de éstas la falta de rigor científico.

Como puede observarse a partir de los enunciados anteriores, sólo en los discursos científicos del tipo monografías de grado se revela una concepción coherente en lo atinente a las características de los componentes del proceso didáctico. Este tipo de discursos se elaboran en el ámbito de la planeación, planificación y evaluación del mismo proceso y por uno de los implicados en él: el estudiante universitario en actividad de práctica docente o de trabajo de investigación que analiza la situación de la enseñanza de la escritura y que, a partir del conocimiento que obtiene mediante el análisis de datos, diseña, aplica y evalúa una propuesta en la que tiene en cuenta los elementos que le aporta la teoría científica, el discurso oficial y la realidad empírica. En nuestro criterio, esta modalidad de construcción del discurso, que aúna teoría y práctica demuestra la coherencia que se logra gracias a esta unidad dialéctica.

Generalmente, en la construcción de los discursos oficial y el científico, se niega la posibilidad de la articulación teoría-práctica a través de la implicación de sus productores en el proceso didáctico, por lo que dichos discursos devienen desarticulados, sin contemplar todos los componentes de la actividad didáctica y desconociendo algunas de las características de los procesos de enseñanza-aprendizaje a los que se refieren.

\section{Bibliografía}

AGUIRRE, M. (1987). “En torno a la expresión poética (1971)”, en Dubsky, J. y otros. Selección de lecturas para redacción. La Habana: Pueblo y Educación, p.p. 129-156.

ALTHUSSER, L. (1974). "Sobre la ideología y el Estado", en Escritos. Barcelona: Laia.

ÁLVAREZ DE ZAYAS, C. M. (1999). La escuela en la vida: Didáctica, tercera edición, La Habana: Pueblo y Educación.

ARMSTRONG, T. (2006). Inteligencias múltiples en el aula: Guía práctica para educadores. Barcelona Paidós Ibérica.

BANGUERO, S. P. (SD) Los niños necesitan maestros que lean y escriban bien. Disponible en: http://aupec.univalle.edu.co/informes/ feb99/lectura.html.

BLOOMFIELD, L. (1964). Lenguaje. Lima, Perú: Universidad Nacional Mayor de San Marcos.

BORJA ALARCÓN, I. y LÓPEZ VEGA, A. (1999) Las palabras en la escuela: Experiencia educativa en el área de castellano. Santafé de Bogotá: Universidad de los Llanos.

BOURDIEU, P. y PASSERON, J. C. (1998). La reproducción: Elementos para una teoría del sistema de enseñanza. México: Fontamara.

BRAVO SIERRA, R. (1996) Investigación social. Madrid: Paraninfo.

CASSASUS, J. (coord.) (2001). Primer estudio internacional comparativo sobre lenguaje, matemática y factores asociados, para alumnos del tercer y cuarto grado de la educación básica. Informe técnico. Laboratorio Latinoamericano de Evaluación de la Calidad de la Educación -UNESCO. Disponible en: http://unesdoc. unesco.org/images/0014/001492/149268s.pdf, 2001.

CISNEROS ESTUPIÑAN, M. (2005). Mejorar los procesos lectoescriturales desde la educación básica. En La didáctica de la lengua materna: Estado de la discusión en Colombia. Santiago de Cali: Universidad del Valle. 
COLOMBIA. MINISTERIO DE EDUCACIÓN NACIONAL. OFICINA ASESORA DE PLANEACIÓN Y FINANZAS, (junio de 2004), El desarrollo de la educación en el siglo XXI. Informe Nacional de Colombia. Disponible en: http:// www.oei.es/quipu/colombia/ibecolombia.pdf,.

\section{CONFERENCIA MUNDIAL DE EDUCACIÓN} PARA TODOS. (1a: 1990: Jomtein) Declaración Mundial sobre Educación para Todos: La satisfacción de las necesidades básicas de aprendizaje. Disponible en: http://www.mineducacion.gov. co/cvn/1665/articles-110467_archivo_pdf.pdf.

CORREA RESTREPO, M. y OROZCO HORMAZA, M. C. (2001) "Dominio gradual de la intencionalidad en la escritura de textos narrativos" en Lectura y escritura para aprender a pensar. Memorias del I Coloquio Internacional y III Regional de la Cátedra UNESCO para la lectura y la escritura en América Latina. Cali : Universidad del Vall'e.

DE MORA, J. M. (Primavera-otoño, 2005). “Milenios de atracción entre la transmisión oral y la escrita", en India. Acta poética 26 (1-2).

DE SAUSSURE, F. (1980). Curso de lingüística general. Madrid: Akal.

DUCKWORTH, E. (SD) "Lenguaje y pensamiento según Piaget", en Coll, C. (comp). Psicología genética y educación : Recopilación de textos sobre las aplicaciones pedagógicas de las teorias de Jean Piaget. Barcelona: Oikos-Tau, p.p. 61-74.

FERREIRO, E. (2001). Alfabetización: Teoría y práctica, Cuarta edición, México: Siglo XXI.

GARTON, A. y PRATT, C. (1991) Aprendizaje $y$ proceso de alfabetización : El desarrollo del lenguaje hablado y escrito, Barcelona: Centro de Publicaciones del Ministerio de Educación y Ciencia.
GEYMONAT, L. (1969). El pensamiento científico.. 4 ed. Buenos Aires: Editorial Universitaria de Buenos Aires.

GOODMAN, K. (agosto. 2003) "El aprendizaje y la enseñanza de la lectura y la escritura”, en Revista Enunciación $\mathrm{N}^{\circ}$ 8. Bogotá: Universidad Distrital Francisco José de Caldas, P.p.77-98.

GORSKI, D. P. (1961) “Lenguaje y conocimiento" en Gorski, D. P. y otros. Pensamiento y lenguaje. Traducción de Augusto Vidal Roger. México: Grijalbo. p. 8-105.

GUZMÁN R., R. J. (1998). “El manejo del lenguaje escrito", en JURADO VALENCIA, F. y BUSTAMANTE ZAMUDIO, G. Los procesos de la escritura: Hacia la producción interactiva de los sentidos. Santafé de Bogotá: Magisterio, p.p. 135-147.

INSTITUTO COLOMBIANO PARA EL FOMENTO DE LA EDUCACIÓN SUPERIOR. La evaluación en lenguaje. Disonible en: http://200.14.205.63:8080/portalicfes/home_2/ rec/arc_3595.pdf.

JAIMES CARVAJAL, G. (2006). "El lenguaje y la comunicación humana", en JAIMES CARVAJAL, G. (coord.). Comprensión y producción de textos orales y escritos en el nuevo sistema acusatorio penal. Bogotá: Consejo Superior de la Judicatura.

JOLIBERT, J. y Grupo de docentes de ECOUEN, (1995). Formar niños productores de textos, sexta edición. Traducción de Viviana Galdames Franco y Alejandra Medina Moreno. Santiago de Chile: Dolmen.

LLINÁS, R. R. (1995). “El reto. Ciencia, educación y desarrollo: Colombia en el siglo XXI", en Misión de ciencia, educación y desarrollo. Colombia: al filo de la oportunidad. Informe conjunto. Santafé de Bogotá: Magisterio. 
PÉREZ ABRIL, M. (2003). Leer y escribir en la escuela: algunos escenarios pedagógicos y didácticos para la reflexión. Bogotá: Instituto Colombiano para el Fomento de la Educación Superior.

ROSENTAL, M. e IUDIN, P. (1975). Diccionario filosófico. Traducción Editora Política. (s. c.) : Combinado poligráfico de Guantánamo 'Juan 'Marinello'.

SECRETARY'S COMMISSION ON ACHIEVING THE NECCESARY SKILLS US. DEPARTAMENT OF LABOR. (1996). "El know-how", Adaptado por Gómez Buendía, Hernando, en GÓMEZ BUENDÍA, H. (comp). Educación. La agenda del siglo XXI. Bogotá: Programa dé las Naciones Unidas para el Desarrollo. Tercer Mundo, p. 223.

SHISHKOVA, T. H. y POPOK, V. K. L: (1989). Estilística funcional. (s. 1.): (s. e.).

SLAKTA, D. (1975). L'ordre du texte En Etude de linguistique appliquée. $\mathrm{N}^{\circ} 19$. Centre d'études du français moderne et contemporain. París: Didier, p.p. $30-42$.
SPIRKIN, A. G. (1961) “Origen del lenguaje y su papel en la formación del pensamiento", en Gorski, D. P. y otros. Pensamiento y lenguaje. Traducción de Augusto Vidal Roger. México: Grijalbo, p.p. 9-67.

TOLCHINSKY LANDSMANN, L. (1993). Aprendizaje del lenguaje escrito: Procesos evolutivos e implicaciones didácticas. Barcelona: Anthropos.

TOLCHINSKY, L. y SIMO, R. (2001). Escribiry leer a través del curriculum. Barcelona: Universidad de Barcelona.

VAN DIJK, T. A. (1980). Estructuras y funciones " del discurso. Bogotá: Siglo XXI. Ideología : Una aproximación multidisciplinaria. Traducción de Lucrecia Berrone de Blanco. Barcelona : Gedisa, 2000. 